\title{
Conformational Effects and Cooperative Interactions in Poly[5(6)-vinylbenzimidazole]-Catalyzed Solvolyses of Anionic, Long-Chain Substrates
}

\author{
C. G. OVERBerger ${ }^{1}$ AND C. J. Podsiadly ${ }^{2}$ \\ Department of Chemistry and the Macromolecular Research Center, The University of Michigan, \\ Ann Arbor, Michigan 48104
}

Received March 1, 1973

\begin{abstract}
A new synthetic procedure for the monomer, 5(6)-vinylbenzimidazole, has been developed. Also, a new method for the formation of the benzimidazole moiety is reported. The monomer, 5(6)-vinylbenzimidazole, was subjected to a free radical solution polymerization and a solid state thermal polymerization. Poly[5(6)-vinylbenzimidazole], prepared by the solution-free radical polymerization, was shown to be a linear addition polymer with pendant benzimidazole groups, whereas the thermally prepared polymer was shown to have a different structure. The solvolyses of negatively charged esters with varying aliphatic chain lengths catalyzed by poly [5(6)vinylbenzimidazole] and compared to monomeric benzimidazole in $40 \% 1$ propanol-water at $26^{\circ} \mathrm{C}$ revealed that the polymer was more efficient and that its activity was a function of the degree of ionization. Neutral-neutral and neutralanionic benzimidazole interactions are suggested. The polymer conformation was found to have a significant effect on the solvolysis reactions.
\end{abstract}

\section{INTRODUCTION}

A synthesis of 5(6)-vinylbenzimidazole was reported earlier (1), but the last step proceeds in very poor yield. The six-step sequence ending in the dehydrochlorination of 5(6)-( $\beta$-chloroethyl)-benzimidazole gave the desired vinylbenzimidazole and its polymer but the last step was not always reproducible. Poly[5(6)-vinylbenzimidazole] was often obtained by the dehydrochlorination of 5(6)-( $\beta$-chloroethyl)-benzimidazole (2). The solvolyses of the neutral substrate, $p$-nitrophenyl acetate catalyzed by poly[5(6)-vinylbenzimidazole] in 30\% 1-propanol-water exhibited unusual results $(2,3)$. The reaction rate of the poly[5(6)-vinylbenzimidazole]-catalyzed reaction increased with increasing $\mathrm{pH}$ more dramatically than the benzimidazole-catalyzed reaction. The solvolyses of the negatively charged esters 4-acetoxy-3-nitrobenzoic acid and sodium 4-acetoxy-3-nitrobenzenesulfonate catalyzed by poly[5(6)-vinylbenzimidazole] were also investigated. The enhanced catalytic activity of the polymer at high $\mathrm{pH}$ values was attributed to a bifunctional catalysis involving both neutral and anionic pendant

\footnotetext{
1 Present address: Department of Chemistry and the Macromolecular Research Center, The University of Michigan, Ann Arbor, MI 48104.

Taken from the dissertation submitted by C. J. Podsiadly in partial fulfillment of the requirements for the degree of Doctor of Philosophy in the Graduate School of The University of Michigan.

Copyright $(\mathcal{C} 1974$ by Academic Press, Inc.

All rights of reproduction in any form reserved.

Printed in Great Britain 
benzimidazole groups (4). The existence of multifunctional interactions in the poly[5(6)-vinylbenzimidazole]-catalyzed solvolyses was further supported by a study of the activation parameters of these reactions and those of benzimidazole, $N$-methylimidazole, imidazole, poly[ $N$-vinylimidazole] and poly[4(5)-vinylimidazole] (5). In order to investigate further the contribution of apolar and electrostatic interactions for the macomolecule-substrate complex formation, the solvolytic reactions of various negatively charged long aliphatic chain esters catalyzed by poly[5(6)-vinylbenzimidazole] (relative to the catalytic activity of the monomeric analog, benzimidazole) were investigated in different compositions of alcohol-water and at different $\mathrm{pH}$ values. The second purpose of this research was to determine whether different polymerization conditions for 5(6)-vinylbenzimidazole lead to polymers of differing structure.

\section{RESULTS AND DISCUSSION}

\section{Synthesis of Monomer}

A new procedure for the synthesis of 5(6)-vinylbenzimidazole has been developed (Fig. 1). The six-step sequence terminates with the decarboxylation of $\beta$-[5(6)-benzimidazole]acrylic acid (VI) which is reproducible and gives yields of $52 \%$. The greatest difficulty in the synthesis was concerned with the transformations of (IV) through (VII).

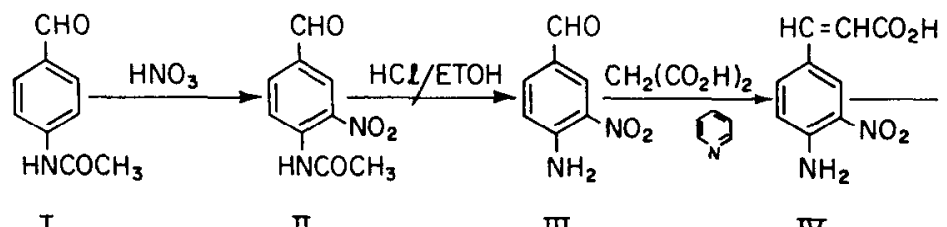

I

II

IV

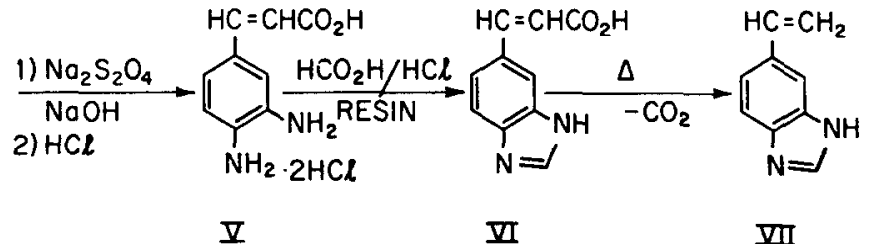

FiG. 1. Synthesis of 5(6)-vinylbenzimidazole.

The reduction of 3-nitro-4-aminocinnamic acid (IV) to 3,4-diaminocinnamic acid (V) by hydrogenation or metallic reduction, under a variety of conditions, was unsuccessful. This transformation was finally carried out by the treatment of a strongly basic solution of (IV) with sodium dithionite, $\mathrm{Na}_{2} \mathrm{~S}_{2} \mathrm{O}_{4}$, followed by acidification (6). The resulting 3,4-diaminocinnamic acid dihydrochloride can be treated with sodium acetale to liberate the free diamine but under these conditions the free diamine is oxidized. The dihydrochloride salt is stable to oxidation and further transformations were made with this compound. 
A variety of methods to effect the ring closure of (V) to form (VI) that have been reported in the literature were attempted (7), but proved to be unsuccessful. The reaction of 3,4-diaminocinnamic acid dihydrochloride with formic acid and $4 \mathrm{~N}$ hydrochloric acid under reflux conditions produced only a trace of $\beta$-[5(6)-benzimidazole]acrylic acid and instead formed polymer. The attempted ring closure under basic conditions led to degradation of the diamine and under milder conditions no reaction took place. Therefore, a new method was developed which involved the use of a polymeric resin. The ring closure using this procedure proceeded successfully at room temperature in $96 \%$ yield. A similar procedure was recently reported utilizing higher temperatures $(8)$.

The diamine dihydrochloride (V) was reacted with formic acid, $4 \mathrm{~N}$ hydrochloric acid and Dowex-50w-x8 (strong sulfonic acid) at room temperature. The mechanism of this ring closure was investigated and proved to be identical with the acid-catalyzed ring closure at reflux temperatures (9). Several reactions to form unsubstituted benzimidazole compounds utilizing o-phenylene diamine were investigated. Two possible mechanisms for the attachment of the diamine and the resulting benzimidazole moiety to the resin were proposed: (a) the formation of a covalent sulfonamide linkage between a sulfonic acid group on the resin and one of the amine groups, or (b) a simple proton transfer from the sulfonic acid to the amine group resulting in a purely electrostatic interaction between the two groups. In order to distinguish between the two suggested mechanisms above, the infrared spectra of the intermediates in the reaction of $o$-phenylene diamine dihydrochloride to benzimidazole were determined. In all experiments the sulfonic acid or sulfonate absorption bands remained unchanged with no sulfonamide absorption bands observed (10). The reactions investigated were similar to the preparation of (VI) and produced benzimidazole in an $80 \%$ yield. The ease of the benzimidazole ring formation is probably due to the high localization of reactants in the domain of the strongly acidic resin.

The sometimes difficult decarboxylation of $\beta$-[5(6)-benzimidazole] acrylic acid (VI) to 5(6)-vinylbenzimidazole (VII) was found to proceed successfully in a sublimation apparatus. This allowed short path distillation of the monomer onto a cold finger condenser as an oil, which subsequently crystallized. The monomer was characterized by its infrared spectrum, nuclear magnetic resonance spectrum, clemental analysis, and melting point.

\section{Synthesis of Polymers}

The monomer, 5(6)-vinylbenzimidazole, was subjected to two different types of polymerization. It polymerized readily in solution using free radical initiation (azobisisobutyronitrile; AIBN), forming a clear viscous liquid in $48 \mathrm{hr}$ from absolute methanol. The polymer was precipitated by addition to anhydrous diethyl ether yielding a white, powdery, insoluble solid in $70 \%$ conversion. A typical sequence in the polymer is illustrated:

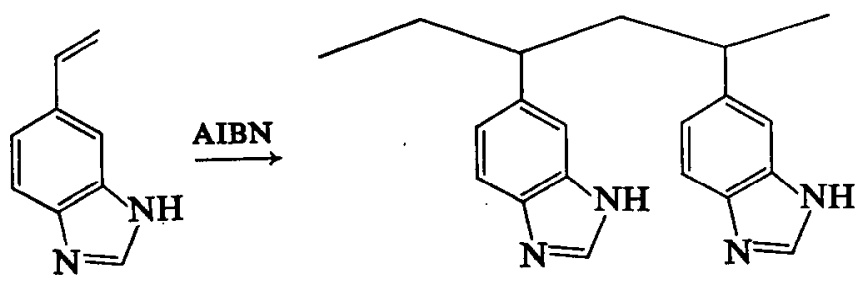


The polymer was insoluble in pure alcohol at room temperature but could slowly be dissolved in hot methanol. It is also insoluble in pure water and nonpolar solvents. The analysis indicated that 1 mole of water was bound to the polymer. This could not be removed by prolonged heating at $100^{\circ} \mathrm{C}$ over phosphorous pentoxide. Similar behavior has been noted for poly [L-histidine] (11) and for poly[4(5)-vinylimidazole] (12). The $\mathrm{p} K$ for the benzimidazole groups in the poly[5(6)-vinylbenzimidazole] (PVBIm) was found to be 4.50 , compared to the $\mathrm{p} K$ of benzimidazole of 5.15 . This lowering of $\mathrm{p} K$ is expected (II).

The monomer also polymerized in a solid state thermal polymerization to a clear yellow film which was insoluble in anhydrous acetone and anhydrous diethyl ether. It was dissolved in methanol-water and precipitated in anhydrous diethyl ether to yield an off-white, powdery, insoluble polymer in $70 \%$ conversion. This polymer was insoluble in pure alcohol and pure water, and was difficultly soluble in $50 \%$ methanolwater. The analysis indicated that no water was bound to the polymer; the $\mathrm{p} K$ for this polymer was 6.90 .

\section{Polymer Characterizations}

It is suggested that 5(6)-vinylbenzimidazole yields polymers of differing structure when the conditions of polymerization are changed. Specifically, a solution, free radical polymerization yielded a linear addition polymer with pendant benzimidazole groups, whereas a solid state thermal polymerization leads to incorporation of the benzimidazole ring system into the polymer backbone. This proposal has been extensively studied and the results tabulated here support the argument (Table 1). In addition to this, the polymer prepared by the solution, free radical technique exhibits unusual structural properties. The structure of the thermal polymer has not been definitely established.

Poly[5(6)-vinylbenzimidazole] (PVBIm) and the thermal polymer differ in their ultraviolet spectra as shown in Table 1. PVBIm exhibits a band at $250 \mathrm{~nm}$ which disappears upon acidification. This band is attributed to the imidazole moiety of the molecule (13). The thermal polymer shows no easily discernible band at $250 \mathrm{~nm}$, possibly due to some interference of the imidazole ring system. Upon acidification the thermal polymer shows a similar behavior to PVBIm. Reagent grade benzimidazole exhibits spectra identical to those for PVBIm.

The infrared spectra do not provide any additional information concerning the structural differences of these polymeric samples because of their similarity. The spectra of the polymers in $\mathrm{KBr}$ indicate a broad band at $2400-3600 \mathrm{~cm}^{-1}$ for $\mathrm{N}-\mathrm{H}-\mathrm{N}$ and sharp bands at 1620 and $1500 \mathrm{~cm}^{-1}$ for $\mathrm{C}=\mathrm{C}$ and $\mathrm{C}=\mathrm{N}$ stretching.

The viscosity measurements were the first indication of an unusual effect operating in the alcohol-water solvent systems. In 50\% 1-propanol-water both polymers show an abnormal viscosity-concentration relationship which strongly suggests extensive aggregation (14) (Fig. 2). This aggregation could occur because of extensive intermolecular H-bonding (15). The intrinsic viscosities, $[\eta]$, are estimated values which are extremely high. With the addition of potassium chloride to a concentration of $0.5 \mathrm{M}$, the viscosity-concentration relationship becomes normal. Intermolecular H-bonding is presumed to be eliminated - this behavior is well precedented in polypeptide chemistry (16). The intrinsic viscosities now obtained show that the thermal polymer is possibly more extended than PVBIm. 


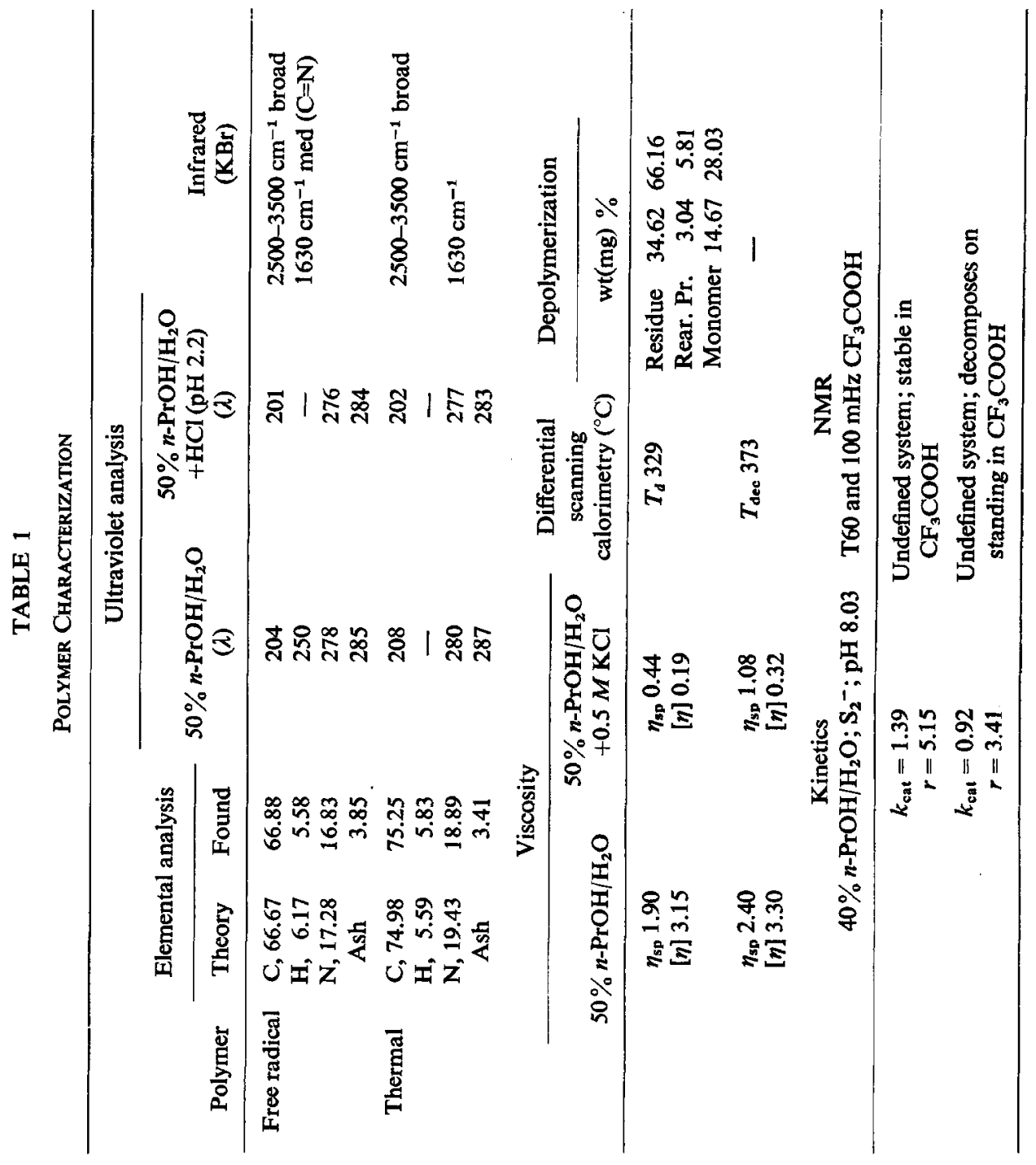



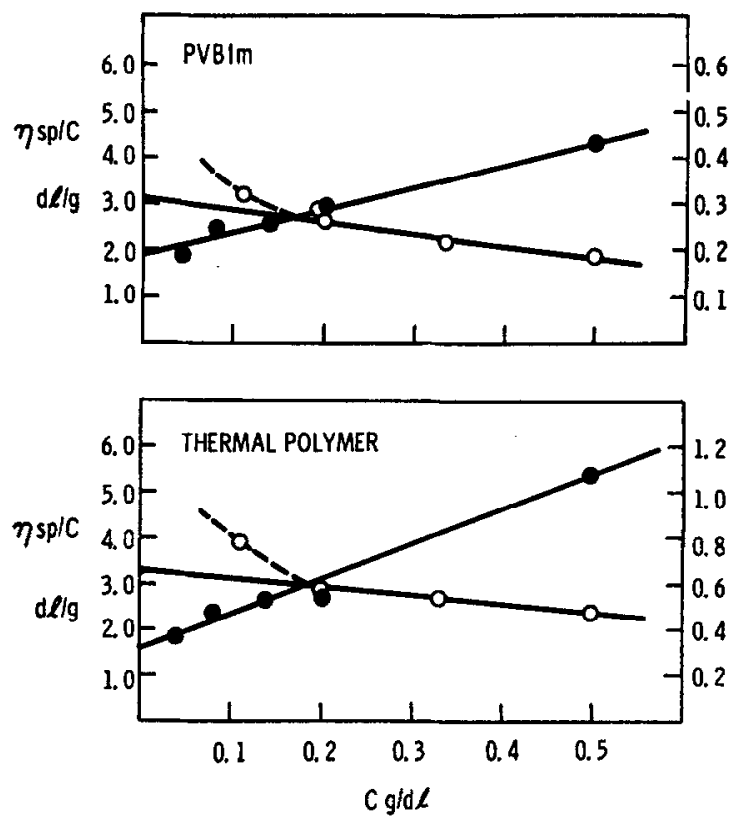

Fig. 2. Viscosity-concentration relationships for poly-5(6)-vinylbenzimidazole and the thermal polymer. $50 \% n-\mathrm{PrOH} / \mathrm{H}_{2} \mathrm{O}(0), 50 \% n-\mathrm{PrOH} / \mathrm{H}_{2} \mathrm{O}+0.5 \mathrm{M} \mathrm{KCl}(\bullet)$.

To study the thermal decomposition of these polymers, differential scanning calorimetry (DSC) was utilized. The DSC curve for PVBIm (Fig. 3) shows a strong endotherm which is postulated as being a "kinetically controlled depolymerization" because of the visual appearance of liquid monomer on the glass cover plate in the instrument. The



FIG. 3. Differential scanning calorimetry curve for poly-5(6)-vinylbenzimidazole. 
DSC curve for the thermal polymer (Fig. 4) shows that this material undergoes a decomposition by the appearance of many peaks with each possibly representing some specific transition (gas evolution). These data alone show that there are some drastic differences in the polymer structures.

The results of a quantitative depolymerization, which are presented in Table 1, verify the observation made in the DSC experiments, namely that some monomer is produced. This result is similar to the degradation of poly(styrene) (17).

The two polymers were tested for catalytic activity in the hydrolysis of 3-nitro-4acetoxy-benzoic acid $\left(\mathrm{S}_{2}^{-}\right)$in the system $40 \%$ 1-propanol-water buffered to $\mathrm{pH} 8.03$ with Tris; $\mu=0.02$. The results show that the thermal polymer has about $66 \%$ of the

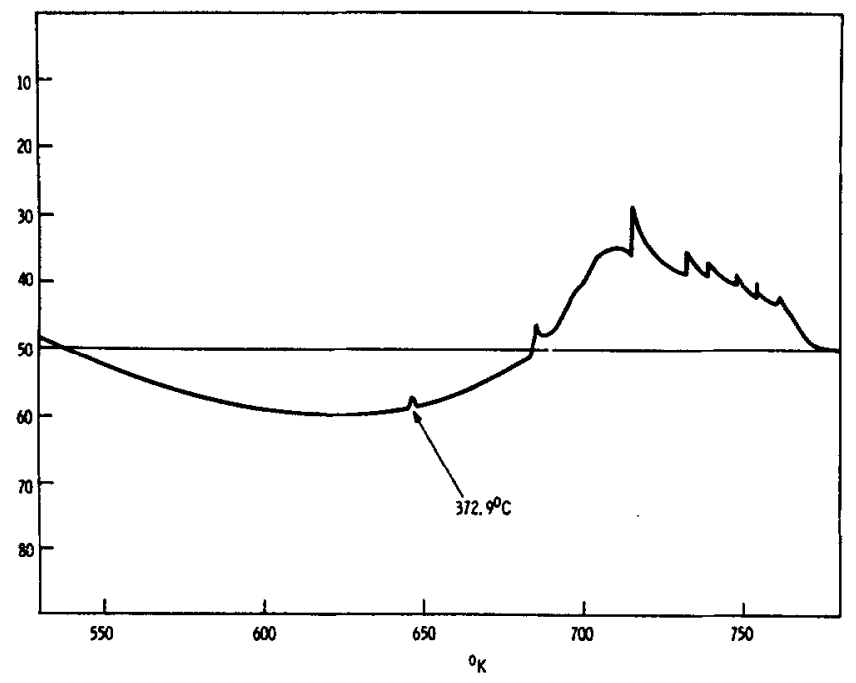

FIG. 4. Differential scanning calorimetry curve for the thermal polymer.

catalytic ability when compared to PVBIm and indicates that similar mechanisms of hydrolysis may be operative.

The nuclear magnetic resonance analyscs are inconclusive even though the initial study was quite extensive. At this time only a few observations can definitely be made. In a T-60 or HA- 100 spectrometer both polymers show no spectra in $50 \%$ deuterated alcohol-water solvents but do exhibit spectra in trifluoroacetic acid. This observation supports the H-bonding proposal and has been documented by Bovey in natural systems (18). The PVBIm shows complete stability in pure trifluoroacetic acid for periods greater than 3 mo whereas the thermal polymer decomposed on standing. This further supports the original proposal, because in PVBIm the benzimidazole rings should be protonated and quite stable and in the thermal polymer protonation of a modified imidazole moiety may lead to rearrangement or decomposition. Lastly, the PVBIm data suggest that this polymer may have a substantial degree of stereoregularity in its structure. To prove this point it is necessary to fractionate carefully a sample of PVBIm utilizing gel permeation chromatography followed by a detailed NMR study of the various fractions. This work is presently being conducted in our laboratories. 
Some data which are not in Table 1 are the results of the potentiometric titrations in $40 \%$ 1-propanol-water at $26^{\circ} \mathrm{C}$ previously referred to. The different $\mathrm{p} K$ values for the two polymers also suggest different structures.

\section{Solvolyses}

The catalytic activities of benzimidazole and poly[5(6)-vinylbenzimidazole] (PVBIm) were investigated by solvolyses of negatively charged esters with varying aliphatic chain length (I).<smiles>[R]C(=O)Oc1ccc(C(=O)O)cc1[N+](=O)[O-]</smiles>

I

$$
\begin{array}{ll}
\mathrm{S}_{2}^{-} & \mathrm{R}=\mathrm{CH}_{3}^{-} \\
\mathrm{S}_{7}^{-} & \mathrm{R}=\mathrm{CH}_{3}\left(\mathrm{CH}_{2}\right)_{5^{-}} \\
\mathrm{S}_{12}^{-} & \mathrm{R}=\mathrm{CH}_{3}\left(\mathrm{CH}_{2}\right)_{10^{-}} \\
\mathrm{S}_{18}^{-} & \mathrm{R}=\mathrm{CH}_{3}\left(\mathrm{CH}_{2}\right)_{16^{-}}
\end{array}
$$

Buffered $40 \%$ 1-propanol-water was used as a solvent throughout this study. The concentrations of the polymers employed in this and subsequent studies are all based on the pendant benzimidazole groups. In the selection of solvent systems the solubility of the polymer was the determining factor. The results obtained previously $(2,3,5)$ were suspect because of limited solubility of the polymer and it was desirable to use the same 1-propanol-water system for comparison. The minimum 1-propanol-water composition which provided complete solubility was found to be $40 \%$. Solvent compositions containing less alcohol led to precipitation of the catalysts.

The catalytic rate constants for this system are summarized in Table 2. All of the values presented are low; therefore, many experiments were undertaken to get a statistical average. In all cases, a minimum of two experiments is reported. The $r$ value, which is a ratio of the catalytic rate constants of PVBIm and benzidimazole, measures the catalytic efficiency of the polymeric catalyst. The polymer was found to be a better catalyst than benzimidazole for all substrates at every $\mathrm{pH}$ investigated, with maximum efficiency occurring at $\mathrm{pH} 8.03$.

The data in Table 2 are represented graphically in Fig. 5, which shows the pH-rate profiles for all four substrates. Proceeding from a low $\mathrm{pH}$ the polymer goes through a maximum rate then a minimum and finally increases at high $\mathrm{pH}$. The point of the maximum cannot easily be explained-it is not near the $\mathrm{p} K_{1}$ of the polymer as is the case for poly[4(5)-vinylimidazole] $(2,4)$. As the polymer increases in negative charge due to an increase in the $\mathrm{pH}$, there should be an electrostatic repulsion between the polymer and the negatively charged substrates, thus decreasing the catalytic efficiency of the polymer. The final rising portion of the curve can be explained by cooperative cffects of ncutral residues and anionic residues attached to the polymer which can overcome any unfavorable electrostatic effects (4). Figure 6 shows the $\mathrm{pH}$-rate profiles for the benzimidazolecatalyzed reactions. The catalytic rate constant increases as the $\mathrm{pH}$ is increased. This is probably due to the formation of anionic benzimidazole species which are more nucleophilic than the neutral species, thus increasing the rate of hydrolysis. 
TABLE $2^{a}$

Catalytic Rate Constants of Benzimidazole AND POLY-5(6)-VINYLBENZIMIDAZOLE WITH Negattvely Charged Long Aliphatic Chain SURSTRATES

$k_{\text {cat }}($ liters/mole min)

\begin{tabular}{|c|c|c|c|c|}
\hline pH & Substrate & BIm & PVBIm & $r^{b}$ \\
\hline 9.90 & $\begin{array}{l}S_{2^{-}}^{-} \\
S_{7}^{-} \\
S_{12}^{-} \\
S_{18}^{-}\end{array}$ & $\begin{array}{l}0.85 \\
0.55 \\
0.58 \\
0.39\end{array}$ & $\begin{array}{l}1.60 \\
0.81 \\
0.68 \\
0.54\end{array}$ & $\begin{array}{l}1.88 \\
1.47 \\
1.17 \\
1.38\end{array}$ \\
\hline 9.00 & $\begin{array}{l}S_{2^{-}}^{-} \\
S_{7^{-}}^{-} \\
S_{12}^{-} \\
S_{18}^{-}\end{array}$ & $\begin{array}{l}0.32 \\
0.12 \\
0.11 \\
0.11\end{array}$ & $\begin{array}{l}0.73 \\
0.44 \\
0.43 \\
0.49\end{array}$ & $\begin{array}{l}2.28 \\
3.67 \\
3.91 \\
4.45\end{array}$ \\
\hline 8.03 & $\begin{array}{l}S_{2^{-}}^{-} \\
S_{7}^{-} \\
S_{12}^{-} \\
S_{18}^{-}\end{array}$ & $\begin{array}{l}0.29 \\
0.09 \\
0.09 \\
0.09\end{array}$ & $\begin{array}{l}1.21 \\
0.60 \\
0.57 \\
0.67\end{array}$ & $\begin{array}{l}4.17 \\
6.67 \\
6.33 \\
7.44\end{array}$ \\
\hline 7.25 & $\begin{array}{l}S_{2}^{-} \\
S_{7}^{-} \\
S_{12}^{-} \\
S_{18}^{-}\end{array}$ & $\begin{array}{l}0.32 \\
0.11 \\
0.12 \\
0.13\end{array}$ & $\begin{array}{l}0.92 \\
0.39 \\
0.39 \\
0.46\end{array}$ & $\begin{array}{l}2.89 \\
3.63 \\
3.25 \\
3.54\end{array}$ \\
\hline
\end{tabular}

a $40 \% \quad n-\mathrm{PrOH} / \mathrm{H}_{2} \mathrm{O} ; \quad[\mathrm{cat}]=5.0 \times 10^{-4} \quad M$; [sub] $=5.0 \times 10^{-5} M ; \mu=0.02 ; T=26^{\circ} \mathrm{C}$.

${ }^{b} r=k_{\text {cat }}^{\mathrm{BVPIm}} / k_{\mathrm{eat}}^{\mathrm{BIm}}$.

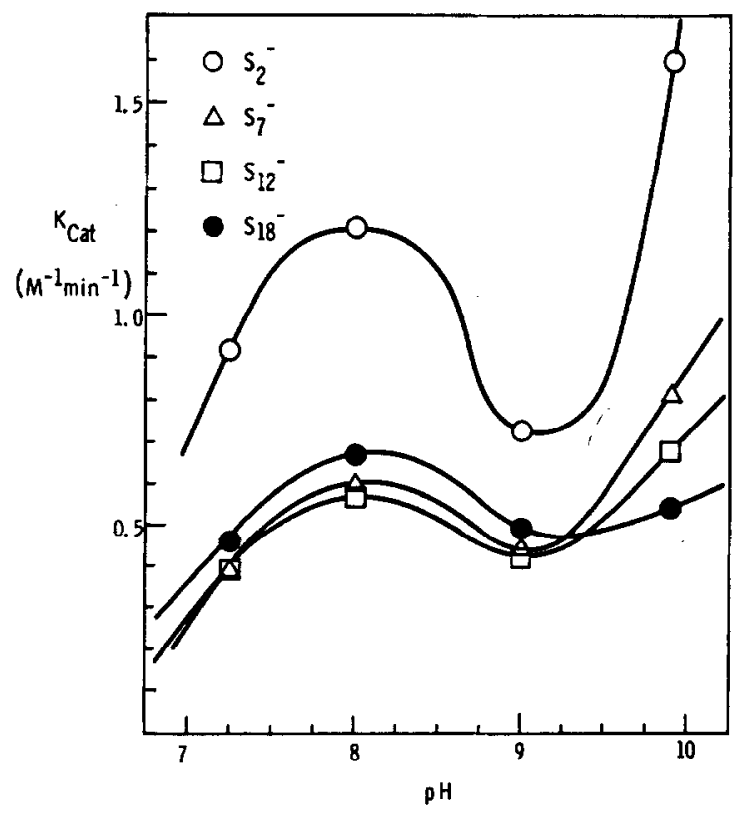

FIG. [5. pH-Rate profiles for the solvolyses of negatively charged long aliphatic chain substrates catalyzed by poly-5(6)-vinylbenzimidazole. $40 \% n-\mathrm{PrOH} / \mathrm{H}_{2} \mathrm{O} ; \mu=0.02$. 


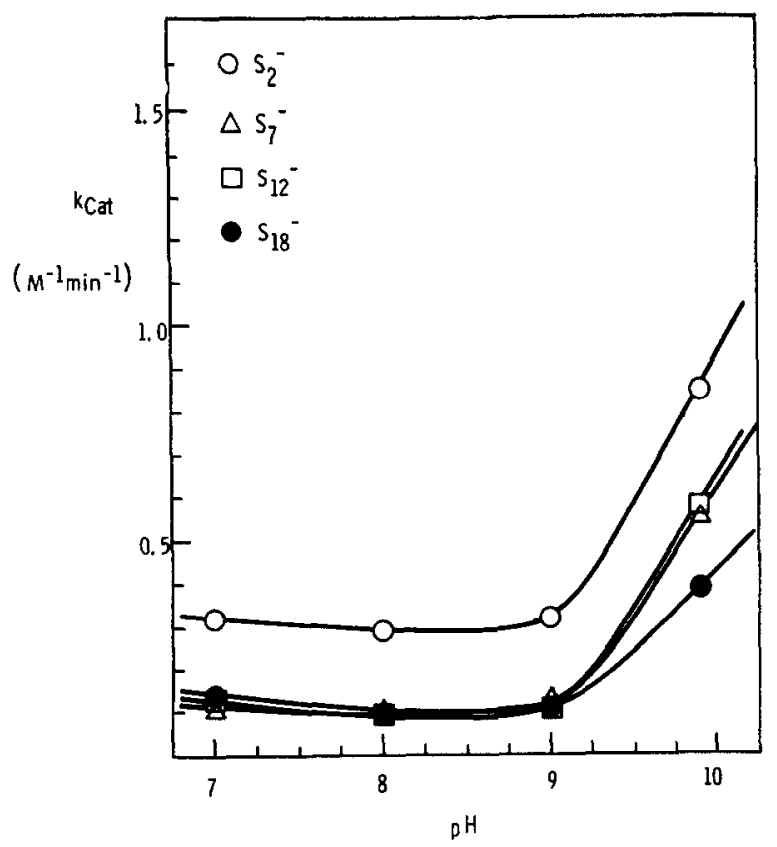

FIG. 6. pH-Rate profiles for the solvolyses of negatively charged long aliphatic chain substrates catalyzed by benzimidazole. $40 \% n-\mathrm{PrOH} / \mathrm{H}_{2} \mathrm{O} ; \mu=0.02$.

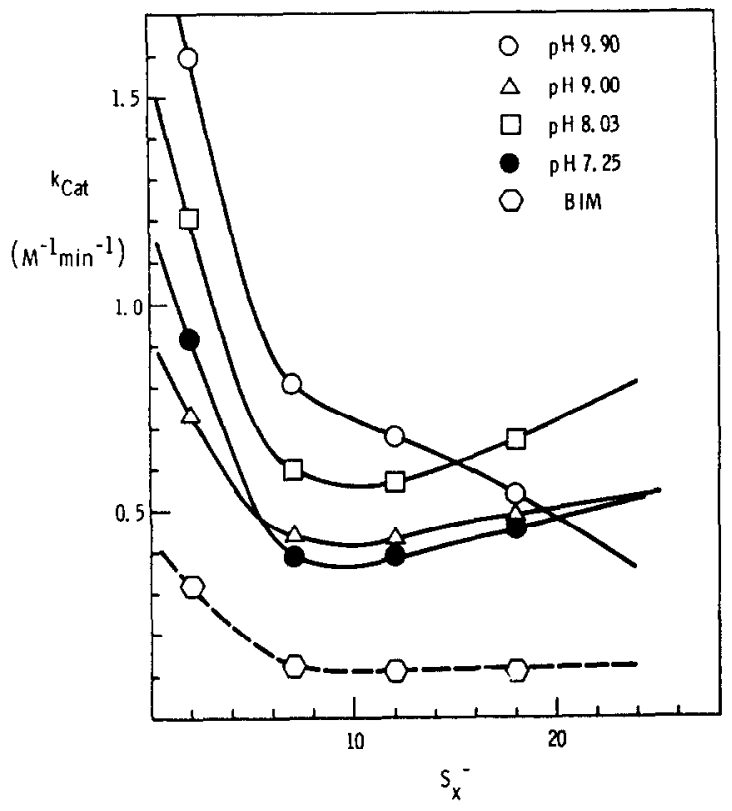

FIG. 7. Effect of varying chain length of negatively charged esters on the catalytic rate constant for poly-5(6)-vinylbenzimidazole catalyzed solvolyses at various $\mathrm{pH} .40 \% n-\mathrm{PrOH} / \mathrm{H}_{2} \mathrm{O} ; \mu=0.02$. 
Figure 7 shows the effect of varying the aliphatic chain length of the substrate on the catalytic rate constants. For benzimidazole we have a representative plot which shows the highest value for $k_{\text {cat }}$ at $\mathrm{S}_{2}{ }^{-}$which decreases and then levels off with increasing size of the substrate. For PVBIm, at pH 10 , the $k_{\text {cat }}$ drops quickly in going from $\mathrm{S}_{2}{ }^{-}$to $\mathrm{S}_{7}{ }^{-}$ and then linearly decreases with increasing size of the substrate. This is an indication that as the size of the negatively charged substrate increases it becomes difficult to encounter the domain of the polymer. The polymer has substantial negative charge at this $\mathrm{pH}$ value due to anionic residues which are more nucleophilic than the neutral residues. If apolar forces would be operative in this system we would expect an increase in rate with a size increase of the substratc, but it appears that these forces are very weak

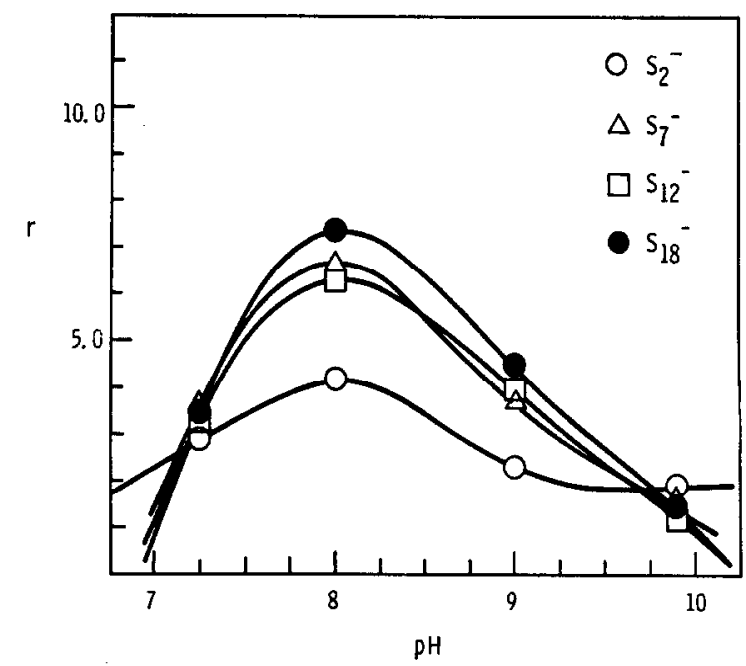

FIG. 8. The effect of $\mathrm{pH}$ on the reactivity ratio, $r$, for the solvolyses of negatively charged long aliphatic chain substrates. $40 \% n-\operatorname{PrOH} / \mathrm{H}_{2} \mathrm{O} ; \mu=0.02$.

in the solvent system used and cannot overcome the strong electrostatic forces. At the other $\mathrm{pH}$ values we have a slight increase with increasing chain length and this may be due to secondary apolar effects. If these data are corrected for the monomeric, benzimidazole catalysis, the true polymeric effects should appear. Figure 8 shows the effect.on the ratio, $r$, with a variation in the $\mathrm{pH}$. The catalytic efficiency of the polymer is greatest at $\mathrm{pH} 8.0$, which corresponds to the maximum in the $\mathrm{pH}$-rate profiles. Also, at this $\mathrm{pH}$ the polymer is more efficient for the substrates with the longer chain lengths, a fact which may be significant with respect to additional attractive forces, i.e., the apolar bond, but the values are still very low.

\section{Viscosity-Reactivity Relationships}

Because of the low catalytic rate constants and the unusual shape of the pH-rate profiles, detailed experiments were carried out to ascertain the viscosity-reactivity relationships of this kinetic system. The results of this study are summarized in Table 3 . In an attempt to duplicate the environment in the kinetic experiments, the identical solutions were used in the viscometer. All the data in Table 3 are corrected. The slope 
TABLE $3^{a}$

Viscosity Measurements of the Solvent Systems Utilized in the Kinetic STUdies

\begin{tabular}{ccccrr}
\hline $\begin{array}{c}\text { Solvent } \\
\text { system }\end{array}$ & $\mathrm{pH}$ & $\begin{array}{c}\eta_{\mathbf{s p}}{ }^{b} \\
(0.5 \mathrm{~g} / \mathrm{dl})\end{array}$ & $\begin{array}{c}{[\eta]} \\
(\mathrm{dl} / \mathrm{g})\end{array}$ & \multicolumn{1}{c}{$k$} & \multicolumn{1}{c}{$k^{\prime}$} \\
\hline $50 \% n-\mathrm{PrOH} / \mathrm{H}_{2} \mathrm{O}$ & - & 0.66 & $0.84^{c}$ & -0.35 & -0.50 \\
$50 \% n-\mathrm{PrOH} / \mathrm{H}_{2} \mathrm{O}$ & - & 0.35 & 0.28 & 0.14 & 1.79 \\
$+0.5 \mathrm{M} \mathrm{KCl}$ & & & & & \\
$40 \% n-\mathrm{PrOH} / \mathrm{H}_{2} \mathrm{O}$ & - & 0.56 & $0.66^{c}$ & -0.20 & -0.46 \\
& 9.90 & 0.31 & 0.27 & 0.09 & 1.25 \\
& 9.00 & 0.33 & 0.26 & 0.14 & 2.03 \\
& 8.03 & 0.36 & $0.42^{c}$ & -0.13 & -0.71 \\
& 7.25 & 0.36 & 0.28 & 0.16 & 2.07 \\
\hline
\end{tabular}

a Poly-5(6)-vinylbenzimidazole; series 150 (K376) Cannon-Fenske viscometer; $T=25 \pm 0.01^{\circ} \mathrm{C}$.

${ }^{b} \eta_{\mathrm{sp}} / C=[\eta]+k^{\prime}[\eta]^{2} \mathrm{C}$.

${ }^{c}$ Estimated intrinsic viscosities.


Fig. 9. Viscosity-concentration relationships for poly-5(6)-vinylbenzimidazole. 
of a plot of the reduced specific viscosity vs concentration is $k$ which can then be transformed into $k^{\prime}$, the Huggins' constant (14). A correlation of $k^{\prime}$ with the conformation of the polymer in solution was attempted, and while the results are quite interesting, specific conclusions cannot be unambiguously made. In 1-propanol-water systems at neutral $\mathrm{pH}$, PVBIm exists as molecular aggregates stabilized by intermolecular $\mathrm{H}$ bonding which can be destroyed with the addition of $0.5 \mathrm{M} \mathrm{KCl}$ (Fig. 9). As the concentration of the polymer is increased, the viscosity decreases, resulting in a negative slope with an intercept that indicates extremely high molecular weight $(14,16)$. On the other



Fig. 10. Viscosity-concentration relationships for poly-5(6)-vinylbenzimidazole at various $\mathrm{pH}$.

hand, by adding salt, which is known to break up aggregates (16), we get a normal viscosity-concentration relationship with a much more accurate interpretation of the intrinsic viscosity. Figure 10 illustrates the results obtained for the buffered systems. At $\mathrm{pH} 7.25,9.00$ and 9.90 , the polymer exhibits a normal viscosity-concentration relationship, while at $\mathrm{pH} 8.03$ an abnormal relationship appears. Figure 11 illustrates the effect of varying the $\mathrm{pH}$ of the concentration independent intrinsic viscosities. Increasing the $\mathrm{pH}$ from 7.25 to 10 results in a definite conformational change which corresponds exactly to the change in the catalytic ability of PVBIm. The inefficiency of this polymer at $\mathrm{pH}$ 8.0, where it probably is in its most favorable conformation for esterolysis reactions, can be readily explained by the reasonable suggestion that intermolecular hydrogen-bonding interferes with the catalytically active pendant benzimidazole groups. 
Also at $\mathrm{pH} 8.0$, the polymer was more efficient for the substrates with the longer chain lengths, a fact which suggested apolar forces even though the effects were small. This can be further supported by the viscosity data. If the polymer exists in a random coil form with intermolecular hydrogen-bonding, the longer chain substrates can be attracted by the hydrophobic portions of the polymer backbone, thereby increasing the effective concentration of substrate around the polymer and thus increasing the effectiveness of the catalyst. Thus, in this sytem we see two effects competing. At $\mathrm{pH} 8$ where we get the maximum catalytic activity for PVBIm, the polymer is aggregated and cannot be



FIG. 11. The effect of varying $\mathrm{pH}$ on the intrinsic viscosity, [ $\eta$ ], of poly-5(6)-vinylbenzimidazole in $40 \% n-\mathrm{PrOH} / \mathrm{H}_{2} \mathrm{O} ; \mu=0.02$.

completely effective. At $\mathrm{pH} 10$ where the polymer begins to extend due to increasing anionic residues, an electrostatic repulsion between a negatively charged substrate and a negatively charged polymer decreases the catalytic activity of the polymer.

To summarize, in the buffered $40 \%$ 1-propanol-water system, poly[5(6)-vinylbenzimidazole] was a better catalyst than benzimidazole in the hydrolysis of various long aliphatic chained negatively charged esters. The polymer showed a maximum of efficiency at $\mathrm{pH}$ 8.0. At this $\mathrm{pH}$ the $\alpha_{1}$ values for benzimidaole and poly[5(6)-vinylbenzimidazole] are 0.913 and 0.982 . The polymer was 7 times more efficient than its monomeric analog for the longer chained substrates and 4 times more efficient for the smallest substrate $\left(\mathrm{S}_{2}{ }^{-}\right)$. We see that under these conditions the benzimidazole groups attached to the polymer chain are more efficient than the monomeric benzimidazole, which is probably due to a cooperative interaction of two neutral benzimidazole groups. That the polymer was more efficient for the longer chained substrates was evidence for additional attractive apolar forces, but as the viscosity studies revealed, the polymer 
aggregates under these conditions thus further reducing its efficiency. We must make these polymers more water soluble to study the apolar effect.

At high $\mathrm{pH}$ values the polymer was twice as efficient as the momomeric benzimidazole species with the smallest substrate $\left(\mathrm{S}_{2}{ }^{-}\right)$and about 1.5 times as efficient with the longer chained species. At these $\mathrm{pH}$ values the polymer has substantial amounts of anionic species and the increased activity is most probably due to the cooperative interactions of a neutral species and an anionic species thus opposing the strong electrostatic repulsive forces.

Based on the evidence presented here, a mechanism which is consistent with the $\mathrm{pH}$ rate profile for the anionic substrates and the known dissociation behavior of the polymer is presented below where (a) at $\mathrm{pH} 8.0$ benzimidazole acts as a nucleophile and then the other neutral benzimidazole function acts as a general base, and (b) at $\mathrm{pH} 10.0$ benzimidazole acts as a nucleophile and then the anion acts as a general base on the tetrahedral intermediate or (c) simple base hydrolysis occurs.<smiles></smiles>

(a)

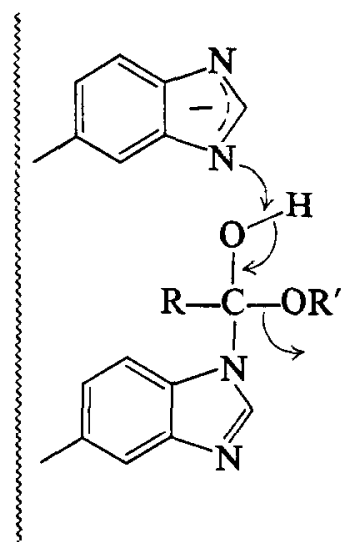

(b)<smiles></smiles>

(c)

In general, the major effect seems to be the electrostatic interactions of the polymer with the negatively charged substrates, and bifunctional catalysis seems to be necessary for the polymer to be effective. These conclusions have also been made for the polymers of 4(5)-vinylimidazole used as catalysts in the esterolysis of various substrates (4).

\section{EXPERIMENTAL METHODS}

All melting points were determined with a Hoover capillary melting point apparatus using open capillary tubes. Melting points are uncorrected. Microanalyses were performed by Spang Microanalytical Laboratory, Ann Arbor, MI, and Galbraith Laboratories, Inc., Knoxville, TN. Infrared spectra were obtained on a Perkin-Elmer Model 237 Spectrometer. Nuclear magnetic resonance spectra were recorded on Varian Models A-60, T-60 and HA-100 spectrometers using tetramethylsilane as internal standard.

3-Nitro-4-acetamidobenzaldehyde (II). This material was prepared in $68 \%$ yield by 
the method of Cohn and Springer (19). Purification was achieved by recrystallization from absolute ethanol yielding long, bright yellow needles; $\mathrm{mp} 151-152^{\circ} \mathrm{C}$.

3-Nitro-4-aminobenzaldehyde (III). A modified procedure of Cohn and Springer (19) was utilized. 3-Nitro-4-acetamidobenzaldehyde ( $20.0 \mathrm{~g} ; 0.096$ mole) was dissolved in $1200 \mathrm{ml}$ of absolute ethanol with $60 \mathrm{ml}$ of concentrated hydrochloric acid and the solution refluxed for $24 \mathrm{hr}$ with stirring. The resulting solution was then cooled to $0^{\circ} \mathrm{C}$ in an ice-water bath. Orange needles which crystallized from the cold acidic ethanol solution were collected, washed with ice-cold ethanol and then suction dried. The solid was then vacuum dried yielding $15.4 \mathrm{~g}(96 \%) \mathrm{mp} 186.5^{\circ} \mathrm{C}\left(190.5-191^{\circ} \mathrm{C}\right)(19)$.

Anal. Calcd for $\mathrm{C}_{7} \mathrm{H}_{6} \mathrm{~N}_{2} \mathrm{O}_{3}(\%): \mathrm{C}, 50.61 ; \mathrm{H}, 3.64 ; \mathrm{N}, 16.86$. Found $(\%): \mathrm{C}, 50.57$; $\mathrm{H}, 3.61 ; \mathrm{N}, 16.89$.

3-Nitro-4-aminocinnamic acid $(I V)$. This material was prepared in $91 \%$ yield by the method of Manecke and Rotter (6). Purification was achieved by recrystallization from water yielding orange crystals; $m p 236-237.5^{\circ} \mathrm{C}$.

3,4-Diaminocinnamic acid (V). The procedure of Manecke and Rotter (6) was utilized with modification. 3-Nitro-4-aminocinnamic acid (9.0 g, 0.0432 mole) was suspended in $300 \mathrm{ml}$ of $3 \mathrm{~N}$ sodium hydroxide and with stirring $45 \mathrm{~g}$ of sodium dithionite $\left(\mathrm{Na}_{2} \mathrm{~S}_{2} \mathrm{O}_{4}\right)$ was added in small portions over a period of $2 \mathrm{hr}$. After complete addition the mixture was left stirring for $3 \mathrm{hr}$. The reduced yellow solution was acidified using $50 \%$ sulfuric acid $(500 \mathrm{ml})$ with external cooling. The precipitated diamine salt was collected by suction filtration and dried. The compound was purified by dissolving the salt in ammonia, treating with Norit, filtered and acidified with gaseous hydrogen chloride. The dihydrochloride salt was collected and dried, yielding $8.8 \mathrm{~g}(81 \%), \mathrm{mp} 225^{\circ} \mathrm{C}$. The free diamine was obtained by treatment of a warm water solution of the salt with sodium acetate. The solution was cooled and the red brown crystals were collected and dried. Yield: $2.0 \mathrm{~g}(26 \%), \mathrm{mp} 157^{\circ} \mathrm{C} .\left(158-159^{\circ} \mathrm{C}\right)(6)$.

$\beta$-[5(6)-Benzimidazole $]$ acrylic acid $(V I)$. The dihydrochloride salt of 3,4-diaminocinnamic acid $(5.0 \mathrm{~g} ; 0.0199$ mole) was suspended in $150 \mathrm{ml}$ of water with $25 \mathrm{ml}$ of formic acid $(88 \%)$. The suspension was placed in a $500 \mathrm{ml}$ round bottom flask along with $25 \mathrm{~g}$ of Dowex-50w-x 8 resin (strong acid resin; washed twice with water) and then mechanically shaken for 3 days at room temperature. The reaction mixture was filtered and the resin was washed throughly with water. The resin was then extracted twice with 150 $\mathrm{ml}$ of $3 \mathrm{~N}$ sodium hydroxidc. The solution was neutralized with hydrochloric acid and allowed to attain room temperature. The neutralized solution was then taken to a constant $\mathrm{pH}$ of 6.5 with hydrochloric acid where a precipitate appeared. The precipitate was filtered, washed with water and dried thoroughly, yield: $3.6 \mathrm{~g}(96.1 \%), \mathrm{mp}$ $270-273^{\circ} \mathrm{C}$ with decomposition. Recrystallization from water yields golden brown crystals $3.1 \mathrm{~g}(82.8 \%), \mathrm{mp} 273-274^{\circ} \mathrm{C}$.

The $\alpha-\beta$ unsaturated carboxylate group was apparent from the infrared and nuclear magnetic spectra with absorption maxima at 1670 and $980 \mathrm{~cm}^{-1}$ and a typical A-B splitting pattern in trifluoroacetic acid $H_{a}, \tau=3.4 ; H_{b}, \tau=2.3$.

Anal. Calcd for $\mathrm{C}_{10} \mathrm{H}_{8} \mathrm{~N}_{2} \mathrm{O}_{2}: \mathrm{C}, 63.82 ; \mathrm{H}, 4.29 ; \mathrm{N}, 14.89$. Found: C, 63.58; $\mathrm{H}, 4.37 ; \mathrm{N}, 14.81$.

5(6)Vinylbenzimidazole (VII). A small sublimator was charged with $\beta$-[5(6)-benzimidazole] acrylic acid $(1.0 \mathrm{~g} ; 0.0053$ mole $)$ and the acid was covered with a small pad of glass wool. The sublimator was evacuated and then immersed into a silicon oil bath 
preheated to $290^{\circ} \mathrm{C}$. The solid begins to decarboxylate $\left(\mathrm{CO}_{2}\right.$ evolution can be followed using a vacuum gauge, but it is better to use a liquid nitrogen trap to condense the $\mathrm{CO}_{2}$ ) and a viscous liquid begins to appear on the probe along with some sublimed acid. The heating was continued until no more $\mathrm{CO}_{2}$ evolution was visible. The material on the probe was then extracted with anhydrous absolute diethyl ether. The ether was removed in vacuo with no heat applied and the viscous oil which remains was left to solidify (3 days), yield $0.398 \mathrm{~g}\left(52^{\circ} \%\right), \mathrm{mp} 110-112^{\circ} \mathrm{C}\left(115-116^{\circ} \mathrm{C}\right)(1)$. Purification was achieved by sublimation; $90^{\circ} \mathrm{C}, 0.05 \mathrm{~mm} ; \mathrm{mp} 110^{\circ} \mathrm{C}$.

The vinyl group was apparent from the infrared and nuclear magnetic resonance spectra with absorption maxima at 910 and $990 \mathrm{~cm}^{-1}$ and a typical ABX splitting pattern in trifluoroacetic acid, $H_{a}, \tau 3.3 ; H_{b}, \tau 4.7 ; H_{c}, \tau 4.3$.

Anal. Calcd for $\mathrm{C}_{9} \mathrm{H}_{8} \mathrm{~N}_{2}: \mathrm{C}, 74.91 ; \mathrm{H}, 5.59 ; \mathrm{N}, 19.43$. Found: C, 74.77, H, 5.66; N, 19.37.

Poly[5(6)-vinylbenzimidazole] (PVBIm). A solution of 5(6)-vinylbenzimidazole $(0.3 \mathrm{~g}$; 0.0021 mole) and $1.7 \mathrm{mg}$ azobisisobutyronitrile $(0.608 \mathrm{~mole} \%)$ in $5.0 \mathrm{ml}$ of absolute methanol was placed in a polymerization tube. The tube was cooled to $-70^{\circ} \mathrm{C}$ and then the mixture was degassed in vacuo. The tube was sealed under vacuum and heated at $75^{\circ} \mathrm{C}$ for $48 \mathrm{hr}$. After cooling to room temperature the mixture was very viscous, yet homogenous. The polymer was precipitated by adding the methanol solution to 500 $\mathrm{ml}$ of absolute diethyl ether, redissolved in methanol-water and reprecipitated in diethyl ether. This procedure was repeated to yield $0.211 \mathrm{~g}$ ( $70 \%$ conversion) of polymer as an off-white powder, $\mathrm{mp} 329^{\circ} \mathrm{C} \mathrm{dec}$.

Anal. Calcd for $\left(\mathrm{C}_{9} \mathrm{H}_{8} \mathrm{~N}_{2} \cdot \mathrm{H}_{2} \mathrm{O}\right)_{x}: \mathrm{C}, 66.7 ; \mathrm{H}, 6.2 ; \mathrm{N}, 17.3$. Found: $\mathrm{C}, 67.9 ; \mathrm{H}, 5.4$; N, 16.8. $[\eta]=0.19(50 \%$ 1-propanol-water $+0.5 \mathrm{M} \mathrm{KCl})$.

Thermal polymerization of 5(6)-vinylbenzimidazole. 5(6)-Vinylbenzimidazole $(0.10184 \mathrm{~g} ; 0.00071 \mathrm{~mole})$ was placed in a vacuum oven for $22 \mathrm{hr}$ at $95^{\circ} \mathrm{C}$. The resulting yellow film was crushed and extracted with anhydrous acetone and anhydrous diethyl ether. The insoluble polymer was collected, dissolved in methanol-water and precipitated in anhydrous diethyl ether. The procedure was repeated to yield $74.35 \mathrm{mg}(73 \%$ conversion) of polymer as an off-white powder, $\mathrm{mp} 373^{\circ} \mathrm{C} \mathrm{dec}$.

Anal. Calcd for $\left(\mathrm{C}_{9} \mathrm{H}_{8} \mathrm{~N}_{2}\right)_{x}: \mathrm{C}, 75.0 ; \mathrm{H}, 5.6 ; \mathrm{N}, 19.4$. Found: C, 75.3; H, 5.8; $\mathrm{N}, 18.9$.

Substrates. The substrates 3-nitro-4-acetoxybenzoic acid $\left(\mathbf{S}_{2}{ }^{-}\right)$, 3-nitro-4-heptanoyloxybenzoic acid $\left(\mathrm{S}_{7}^{-}\right)$, 3-nitro-4-dodecanoyloxybenzoic acid $\left(\mathrm{S}_{12}^{-}\right)$and 3-nitro-4-octa deconoyloxybenzoic acid $\left(\mathbf{S}_{18}^{-}\right)$were kindly provided by $\mathbf{P}$. H. Vandewyer. The syntheses of these substrates have previously been reported (20).

Polymer characterization. Poly[5(6)-vinylbenzimidazole] and the thermal polymer were subjected to characterization. Table 1 shows the data obtained in the attempted structure elucidation of these two polymers. The ultraviolet spectra in $50 \% 1$-propanolwater solutions of PVBIm $\left(4.63 \times 10^{-5} M\right)$, the thermal polymer $\left(6.94 \times 10^{-5} M\right)$ and in $50 \%$ 1-propanol-water $+0.5 \mathrm{M} \mathrm{HCl}(\mathrm{pH} 2.2)$ solutions of PVBIm $\left(4.48 \times 10^{-5} \mathrm{M}\right)$, thermal polymer $\left(6.72 \times 10^{-5} M\right)$, were taken at room temperature utilizing a PerkinElmer Model 402 spectrometer. The infrared spectra were carried out on $\mathrm{KBr}$ pellets of the polymer with medium scan at normal slit width. All viscosity measurements were made using a Cannon-Fenske viscometer, series 150 (K376) at $25 \pm 0.01^{\circ} \mathrm{C}$. Six readings were made for each concentration and the average value used in the subsequent 
calculations. A plot of $\eta_{\mathrm{sp}} / c$ versus concentration $(\mathrm{g} / \mathrm{dl})$ was made for each run. The two polymers were also subjected to thermal analysis using a Perkin-Elmer Model DSC-1B differential scanning calorimeter. PVBIm (4.34 mg) and the thermal polymer $(1.23 \mathrm{mg})$ were weighed in aluminum sample pans and then capped using a crimper to seal a cover onto the sample pan. The prepared samples were then placed in the sample holder along with a reference sample cup and measured at a scan speed of $10 \mathrm{~K} / \mathrm{min}$. The resulting spectra are shown in Figs. 3 and 4. A quantitative depolymerization of PVBIm was attempted. A small sublimator was charged with $52.33 \mathrm{mg}$ of PVBIm and then evacuated for $2 \mathrm{hr}$. The sublimator was then immersed in a sand bath and the temperature was slowly raised to $360^{\circ} \mathrm{C}$ and held there for $2 \mathrm{hr}$. The material on the probe was then extracted with anhydrous diethyl ether, filtered and then the ether was removed invacuo to yield an oil which solidified on standing. The results are shown in Table 1 . The two polymers were tested for catalytic activity in the hydrolysis of 3-nitro-4-acetoxybenzoic acid $\left(\mathrm{S}_{2}{ }^{-}\right)$. The system was $40 \% 1$-propanol-water buffered to $\mathrm{pH} 8.03$ with Tris, ionic strength, $\mu, 0.02$. The results show that the thermal polymer has about $66 \%$ of the catalytic activity when compared to PVBIm. The first attempts at nuclear magnetic spectroscopy were completed utilizing 50\% deuterated methanol-water as a solvent. The solutions produced were viscous and when carried out in the T- 60 spectrometer under a variety of conditions produced absolutely no spectra. The samples were run in a HA-100 spectrometer with similar results. Trifluoroacetic acid was added to these samples in an effort to reduce the viscosity and record a spectra.

Potentiometric titrations. In $15 \mathrm{ml}$ of $40 \% 1$-propanol-water was dissolved enough sample to adjust the concentration to $10^{-4} M$. The ionic strength was adjusted with hydrochloric acid to give $\mu=0.02$ at the end point of titration. By using a microburet and a Beckman Expandomatic pH meter, the solutions were titrated with $1 \mathrm{~N}$ sodium hydroxide at $26^{\circ} \mathrm{C}$ with stirring. The blank titration curves were obtained in a similar manner. Differential titration curves were derived graphically $(21,22)$ from which the degree of dissociations were evaluated. The $\mathrm{pH}-\alpha_{1}$ relationship was found to follow the modified Henderson equation (Eq. 1) (22-24) where $\alpha_{1}$ is the fraction of neutral benzimidazole groups.

$$
\mathrm{pH}=\mathrm{p} K_{1}-n \log \left(\frac{1-\alpha_{1}}{\alpha_{1}}\right) .
$$

The $\mathrm{p} K$ values were evaluated graphically. Benzimidazole was titrated in the same manner. The obtained $\mathrm{p} K$ values are 5.15 for benzimidazole, 4.50 for PVBIm and 6.90 for the thermal polymer.

Kinetic measurements. Kinetic measurements were performed as previously described (2). The solvent, $40 \% 1$-propanol-water, was used for every kinetic run. The solutions were buffered with tris(hydroxymethyl)aminomethane (Tris) $(0.02 M)$-hydrochloric acid at pH 7-9 and with 3-( $N, N$-dimethylamino)propanol $(0.02 M)$-hydrochloric acid at $\mathrm{pH}$ above 9 . The ionic strength was adjusted by addition of potassium chloride. The rates of solvolyses were determined by following the increase in the absorbance of the released phenols at $400 \mathrm{~nm}$. All the obtained data followed first-order kinetics. The pseudo-first-order rate constants $k_{\text {measd }}$ (with catalyst), $k_{\text {blank }}$ (without catalyst), were treated by the expressions $k_{\text {obsd }}=k_{\text {measd }}-k_{\text {blank }}$ and $k_{\text {cat }}=k_{\text {obsd }} /$ [catalyst] where [catalyst] is the molar concentration of benzimidazole functions. 


\section{ACKNOWLEDGMENT}

The authors acknowledge the financial support of the National Institutes of Health under Grant No. 2 RO1 GM 15256, and the Macromolecular Research Center of The University of Michigan. We also wish to thank Professors J. E. Mark and D. T. Longone for their helpful discussions.

\section{REFERENCES}

1. C. G. Overberger, B. Kosters, and T. St. Pierre, J. Polym. Sci., Part A-1 5, 1987 (1967).

2. C. G. Overberger, T. St. Pierre, N. Vorchheimer, J. Lee, ANd S. Yaroslavsky, J. Amer. Chem. Soc. 87, $296(1965)$.

3. C. G. Overberger, T. St. Pierre, and S. Yaroslavsky, J. Amer. Chem. Soc. 87, 4310 (1965).

4. C. G. Overberger AND J. C. Salamone, Accounts Chem. Res. 2, 217 (1969), and references cited therein.

5. C. G. Overberger, T. St. Pierre, C. Yaroslaysky, and S. Yaroslavsky, J. Amer. Chem. Soc. 88, 1184 (1966).

6. G. MANACKe AND U. RotTer, Makromol. Chem. 127, 264 (1969).

7. For a review see J. B. Wright, Chem. Rev. 48, 397 (195i).

8. V. I. Isaguliyants and N. M. ANufrieva, J. Appl. Chem. USSR 44, 1196 (1971).

9. M. A. Phillips, J. Chem. Soc. 1409 (1930).

10. Y. Tanaka and Y. Tanaka, Chem. Pharm. Bull. 13, 399 (1965).

11. A. Patchornik, A. Berger, and E. Katchalski, J. Amer. Chem. Soc. 79, 5227 (1957).

12. C. G. OVerberger and N. VorchHeimer, J. Amer. Chem. Soc, 85, 949 (1963).

13. K. HofmanN, "The Chemistry of Heterocyclic Compounds: Imidazole and its Derivatives," Part I. Wiley (Interscience), New York, 1953.

14. M. L. Huggins, J. Amer. Chem. Soc. 64, 2716 (1942).

15. H. Morawetz, "High Polymers," Vol. 21, Chap. 8. Wiley (Interscience), New York, 1965.

16. N. Timasheff AND G. D. FASMAN, "Structure and Stability of Biological Macromolecules." Dekker, New York, 1969.

17. F. W. Billmeyer, JR., "Textbook of Polymer Science," pp. 354-357. Wiley (Interscience), New York, 1965.

18. F. A. BoveY, "Polymer Conformation and Configuration." Academic Press, New York, 1969.

19. P. Cohn AND L. SPRinger, Monatsh. Chem. 24, 94 (1903).

20. C. G. Overberger, R. C. Glowsky, AND P. H. VANDewyer, J. Amer. Chem. Soc., 95, 6008(1973).

21. T. V. Parke and W. W. Davis, Anal. Chem. 26, 642 (1954).

22. T. Alfrey and H. S. Pinner, J. Polym. Sci. 13, 533 (1954).

23. A. Katchalsky and I. R. Miller, J. Polym. Sci. 13, 57 (1954).

24. H. L. Wagner AND F. A. Long, J. Phys. Colloid Chem. 55, 1912 (1951). 